\title{
Signal Travel Time Anomaly between Earth and Solar Planets
}

\section{Faiçal Ramdani}

Rabat, Morocco

Email: faicalramdani@gmail.com

How to cite this paper: Ramdani, F. (2021) Signal Travel Time Anomaly between Earth and Solar Planets. Journal of High Energy Physics, Gravitation and Cosmology, 7, 14721476.

https://doi.org/10.4236/jhepgc.2021.74089

Received: August 20, 2021

Accepted: October 18, 2021

Published: October 21, 2021

Copyright $\odot 2021$ by author(s) and Scientific Research Publishing Inc. This work is licensed under the Creative Commons Attribution International License (CC BY 4.0).

http://creativecommons.org/licenses/by/4.0/

(c) (i) Open Access

\begin{abstract}
Travel time anomaly in the communicatin of spatial instruments installed in solar planets and Earth are investigated in a model of gravity impact on radio signal propagation. Resulting travel times anomaly shows variations less than $10^{-3}$ seconds to and from Venus and Mars while Sun provides anomaly travel times of about $-2 \times 10^{-2} \mathrm{sec}$ as backward signal needs more times than emitted signal from Earth. In the current explorations on Mars, the travel time anomaly may reach $1.8 \times 10^{-4} \mathrm{sec}$ as orbital Mars position pass through its minimum distance with respect to Earth. Implications of the difference between one and two-way travel times may be related to redshift/blueshift while travel time of received signal is less or greater than emitted signal.
\end{abstract}

\section{Keywords}

Radio Signal, Travel Times, Gravity, Solar Planets, Redshift

\section{Introduction}

Anomaly in space recording data has been observed such as Pioneer anomaly which may have a gravitational origin [1], and has been explained in term of conformal gravity [2]. Galaxie clusters of high magnitude of redshift $(>2)$ were observed [3] [4] while very small scale redshift within solar system cannot be easily obtained. Solar gravitational redshift is suggested from photon and electrons interactions [5]. Many explorations on Mars are now experienced by several institutions to record new data. Nasa perseverance [6] and Chinese Zhurong [7] reached Mars surface in June 2021 and they send and received signals from and to Earth. These experiences are now worked while distance to Mars is at its minimum relative to Earth. It is then an opportunity to examine the travel times of signals based on UHF or X-band in one or two ways. Based on gravity impact 
on velocity of light [8] variations in travel times may be caused as gravity field vary on Mars and solar planets with respect to Earth. Changes in signal frequency and Doppler effect that also contribute to redshift are not addressed. The estimation of changes in travel times of transmitted signals based on velocity variations is here addressed between Earth and planets of the solar system.

\section{Data and Results}

Data from photon travel times inside solar planets are based on initial velocity acquired by photons in a particular planet gravity field. Distances to Earth from Moon and Mars and other solar planets provide both maximum and minimum values related to orbital positions [9]. While radio signal traveltimes emitted from Earth will be received on Mars by Tem $=\mathrm{D} / \mathrm{Ce}$, where Ce is initial velocity acquired on Earth to Mars on one forward way (Table 1). The signal traveltime from Mars to Earth will be then $\mathrm{Tme}=\mathrm{D} / \mathrm{Cm}$, where $\mathrm{Cm}$ is velocity dependant Mars gravity [8]. Two way travel times of a signal emitted to Mars and back to Earth then will be $[\mathrm{Tem}+\mathrm{Tme}]$. Resulting variation $\Delta \mathrm{T}$ [Tem $-\mathrm{Tme}]$ are shown in Figure 1 in addition to travel time estimates of light from Earth to Sun, Venus and Moon

Table 1. Travel time estimates of signals between solar system planets and Earth based on initial velocity acquired by their specific planet gravity field. Variation in the emitted and received signals are indicated.

\begin{tabular}{|c|c|c|c|c|c|c|}
\hline Planet Orbit & $\begin{array}{l}\text { Distance to Earth } \\
\qquad\left[10^{9} \cdot \mathrm{m}\right]\end{array}$ & $\begin{array}{c}\mathrm{T}_{1} \text { from Earth } \\
{[\mathrm{s}]}\end{array}$ & $\begin{array}{l}\mathrm{T}_{2} \text { to Earth } \\
{[\mathrm{s}]}\end{array}$ & $\begin{array}{c}\mathrm{T}_{1}-\mathrm{T}_{2} \\
{[\mathrm{~s}]}\end{array}$ & $\begin{array}{c}\mathrm{T}_{1}+\mathrm{T}_{2} \\
\quad[\mathrm{~s}]\end{array}$ & $\begin{array}{c}\mathrm{T}_{1}+\mathrm{T}_{2} \\
{[\mathrm{mn}]}\end{array}$ \\
\hline Mars-min & 55.7 & 185.795201 & 185.7950096 & 0.000191416 & 371.5902106 & 6.193170177 \\
\hline Mars-max & 401.3 & 1338.592714 & 1338.591335 & 0.001379087 & 2677.184049 & 44.61973415 \\
\hline Mars-average & 227.943824 & 760.3387541 & 760.3379707 & 0.00078334 & 1520.676725 & 25.34461208 \\
\hline Sun & 149.5978707 & 499.0047838 & 499.0269644 & -0.022180575 & 998.0317482 & 16.63386247 \\
\hline Moon min & 0.357 & 1.19082382 & 1.190822172 & $1.64759 \mathrm{E}-06$ & 2.381645992 & 0.0396941 \\
\hline Moon max & 0.407 & 1.357605867 & 1.357603989 & $1.87835 \mathrm{E}-06$ & 2.715209857 & 0.045253498 \\
\hline Venus min & 38.2 & 127.4214844 & 127.4214646 & $1.97955 \mathrm{E}-05$ & 254.8429489 & 4.247382482 \\
\hline Venus max & 261 & 870.6022885 & 870.6021532 & 0.000135252 & 1741.204442 & 29.02007403 \\
\hline Mercure-max & 77.3 & 257.8450456 & 257.8447799 & 0.000265678 & 515.6898255 & 8.594830425 \\
\hline Mercure-min & 221.9 & 740.1787272 & 740.1779646 & 0.000762664 & 1480.356692 & 24.67261153 \\
\hline Jupiter max & 968.1 & 3229.234006 & 3229.241219 & -0.007213733 & 6458.475225 & 107.6412537 \\
\hline Jupiter min & 588.5 & 1963.0247 & 1963.029085 & -0.004385169 & 3926.053786 & 65.43422976 \\
\hline Saturn-min & 1195.5 & 3987.758758 & 3987.758206 & 0.000552022 & 7975.516964 & 132.9252827 \\
\hline Saturn-max & 1658.5 & 5532.160519 & 5532.159753 & 0.000765812 & 11064.32027 & 184.4053379 \\
\hline Uranus-max & 2581.9 & 8612.291374 & 8612.289745 & 0.00162885 & 17224.58112 & 287.076352 \\
\hline Uranus-min & 3157.3 & $10,531.61918$ & $10,531.61719$ & 0.001991854 & 21063.23636 & 351.0539394 \\
\hline Neptune average & 4496.6 & $14,999.0431$ & $14,999.04607$ & -0.002966864 & 29998.08918 & 499.9681529 \\
\hline Pluto-min & 4284.7 & $14,292.22079$ & $14,292.19885$ & 0.021939411 & 28584.41963 & 476.4069939 \\
\hline Pluto-max & 7528.7 & $25,113.04004$ & $25,113.00149$ & 0.038550013 & 50226.04152 & 837.100692 \\
\hline
\end{tabular}




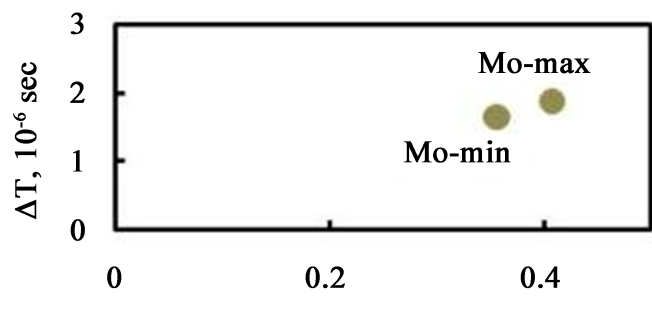

(a)

Distance, $10^{9} . \mathrm{m}$

Moon
Venus
Mercure
Mars
Jupiter
Saturne
Neptune
Uranus
Pluto
Sun
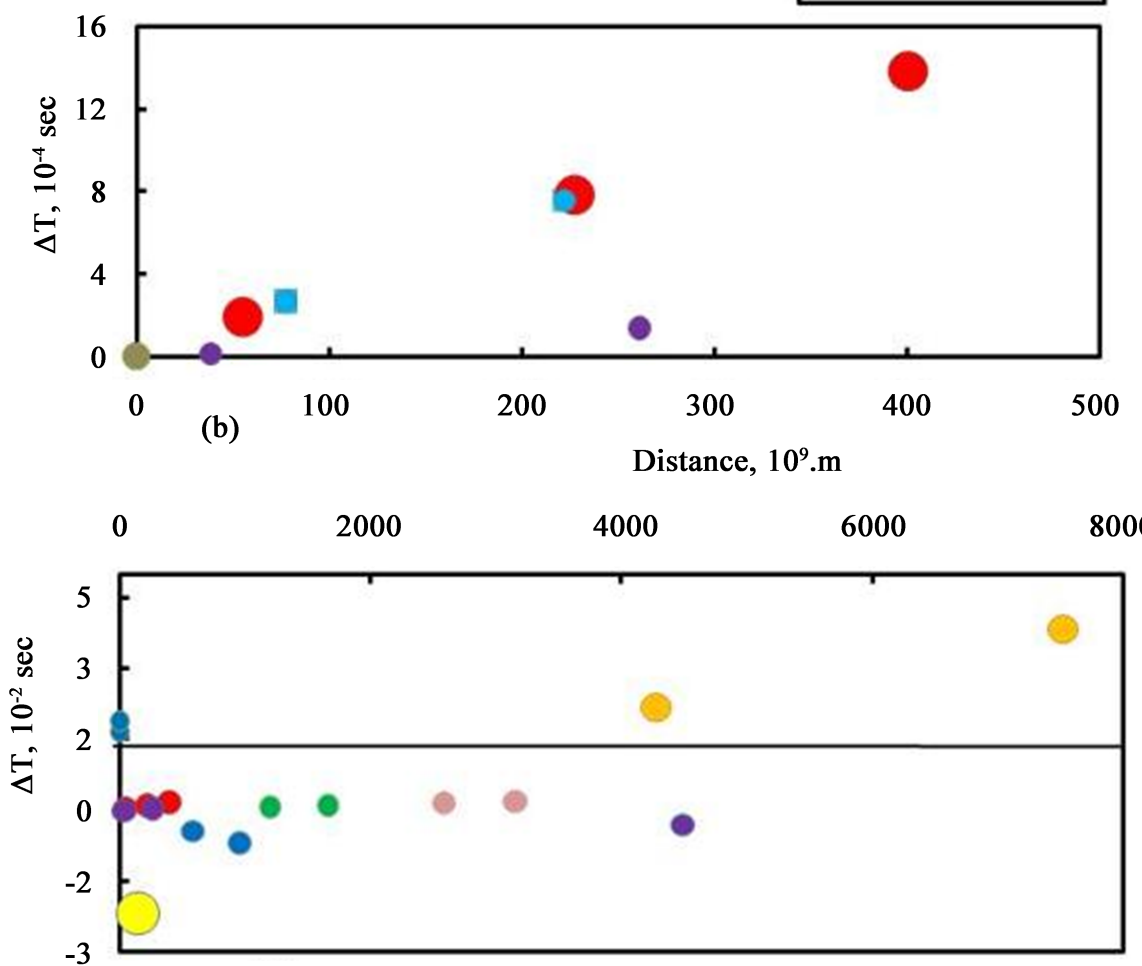

(c)

Figure 1. Relative signal travel time variations $\Delta \mathrm{t}$ versus varying range of distances to Earth is less than $10^{-6} \mathrm{sec}$ between forward and backward signal Earth-Moon (a). This value reaches about $10^{-4} \mathrm{sec}$ forsome solar planets according to both their minimum and maximum orbital distances to earth (b). Itincreases by more than 20 time to about $10^{-2}$ when gravity of Sun and Pluto are considered (c).

and other solar planets. At short distance as between maximum and minimum distances to the Moon the travel time variation is about $10^{-6} \mathrm{sec}$ (Figure 1(a)). Maximum and minimum values at intermediate distances (Figure 1(b)) are in the range of $10^{-3}$ to $10^{-4} \mathrm{sec}$ (Mars, Venus, Uranus). While this variations increased to \pm 0.02 seconds from travel times to Sun and Pluto due to their respective gravity (Figure $1(\mathrm{c})$ ). Figure $1(\mathrm{c})$ shows that other solar planets seem not significant in the travel time difference between forward and backward signals compared to Pluto and Sun. Doppler effect may be observed due to orbital position of planets during travel times between forward and backward signals. In this model, the Doppler effect is not considered in the two way radio signal but 
Table 2. Spectral effect $\mathrm{z}$ estimated in term of velocity from solar planets and Earth based on planet gravity related by assuming stationary of planets during two way travel times of an emitted signal from earth.

\begin{tabular}{cccc}
\hline Planet & $\mathbf{V}(\mathrm{g}), \mathbf{m} / \mathbf{s}$ & $\mathbf{z} \cdot 10^{-4}$ & Spectrum effect \\
\hline Earth & $299,792,458$ & 0 & - \\
Sun & $299,779,132.9$ & 0.44 & redshift \\
Mars/Mercure & $299,792,766.9$ & -0.01 & blueshift \\
Venus & $299,792,504.6$ & -0.0015 & blueshift \\
Jupiter & $299,791,788.3$ & 0.023 & redshift \\
Saturn & $299,792,499.5$ & -0.0013 & blueshift \\
Uranus & $299,792,514.7$ & -0.0005 & blueshift \\
Neptune & $299,792,398.7$ & 0.002 & redshift \\
Pluto & $299,792,918.2$ & -0.015 & blueshift \\
Moon & $299,792,872.8$ & -0.013 & blueshift \\
\hline
\end{tabular}

rather only the changes in velocity acquired by the gravity environment. Table 2 shows $\mathrm{z}$ factor of both red and blueshift which is assumed to represent $(1-\mathrm{Vg} / \mathrm{Vc})$, where $\mathrm{Vg}$ is the photon velocity in a particular gravity field while $\mathrm{Vc}$ is this light velocity on Earth. Negative values of $\mathrm{z}$ represent spectral blueshift obtained for signal reflected from Venus, Mars, Uranus, Pluto and Mercure. Positive $\mathrm{z}$ represents redshift that correspond to signals coming from Sun, Jupiter and Neptune. Both shifted values are separated by 0-line shown in Figure 1(c). High magnitude shifting is perceptible to signals directed to Pluto and Sun.

\section{Discussions and Conclusions}

Distance to Earth also has a moderate impact on travel time difference. Signals coming from Sun and Mars are affected by Earth's gravity in the last $100 \mathrm{~km}$ to reach Earth. As $100 \mathrm{~km}$ appears negligible compared to distance to Mars and Sun the changes will be very low to consider. Signal propagation also may be affected by electrical charges due to solar wind that may reduce the vacuum character of the space between solar planets. At greater distance outside the solar system, the distance seems not to play a critical role but rather the state of gravity of the planet from which the signal is emitted. Assuming that the signal has been not reflected by another planet which transmits to it the speed related to its gravity field. Distance travelled by Planets during the two-way travel times is also small to consider. Maximum one way travel times to Pluto is about 6 hours. During this time Pluto's distance to Earth varies. This variation in distance is an additional factor as Doppler effects contributing to blueshift. Moon, Mars and Venus are at a shorter distance to Earth where variation in distance to Earth during signal travel times may be reduced and the critical factor contributing to blueshift is due to gravitational velocity of the photons. In this model, the space time referential is the solar system with varying local gravity field related planets. 
The expansion of universe is here considered as the solar system is moving as a whole stationary system. The current presence of in situ measurements in Mars may be an opportunity to explore the validity of changes in travel times of radio signals due to velocity gravity dependent in solar system.

\section{Conflicts of Interest}

The author declares no conflicts of interest regarding the publication of this paper.

\section{References}

[1] Ioro, L. (2007) Jupiter, Saturn and the Pioneer Anomaly: A Planetary-Based Independent Test. Journal of gravitational Physics, 1, 1, 5-8.

[2] Varieschi, G.U. (2012) Conformal Cosmology and the Pioneer Anomaly. Physics Research International, 2012, Article ID: 469095. https://doi.org/10.1155/2012/469095

[3] Miley, G. and De Breuck, C. (2008) Distant Radio Galaxies and Their Environment. The Astronomy and Astrophysics Review, 15, 67-144. https://doi.org/10.1007/s00159-007-0008-Z

[4] Miller, T.B, Chapman, S.C., Aravena, M., et al. (2018) A Massive Core for a Cluster of Galaxies at a Redshift of 4.3. Nature, 556, 469-472. https://doi.org/10.1038/s41586-018-0025-2

[5] Trinchera, A. (2020) Redshift Anomaly on the Solar Disk as Multiple Interactions between Photons and Electrons. Journal of High Energy Physics, Gravitation and Cosmology, 7, 1-51. https://doi.org/10.4236/jhepgc.2021.71001

[6] https://nasa.gov/perseverence

[7] Wan, W.X., Wang, C., Li, C.L. and Wai, Y. (2020) Chinas's First Mission to Mars. Nature Astronomy, 4, 721. https://doi.org/10.1038/s41550-020-1148-6

[8] Ramdani, F. (2019) Gravity Constraints on the Measurements of the Speed of Light. International Journal of Astronomy and Astrophysics, 9, 265-273. https://doi.org/10.4236/ijaa.2019.93019

[9] https://nssdc.gsfc.nasa.gov 